\title{
Intrinsic Insular-Frontal Networks Predict Future Nicotine Dependence Severity
}

\author{
니-Ming Hsu, ${ }^{1 \star}$ Robin J. Keeley, ${ }^{1 \star}$ Xia Liang, ${ }^{2}$ 이uia K. Brynildsen, ${ }^{1}$ Hanbing Lu, ${ }^{1}$ Yihong Yang, ${ }^{1}$ and Elliot A. Stein ${ }^{1}$ \\ ${ }^{1}$ Neuroimaging Research Branch, National Institute on Drug Abuse, Intramural Research Program, Baltimore, Maryland 21224, and ${ }^{2}$ Research Center of \\ Basic Space Science, Harbin Institute of Technology, Nangang Qu, Haerbin Shi 150001, Heilongjiang Sheng, People’s Republic of China
}

Although $60 \%$ of the US population have tried smoking cigarettes, only $16 \%$ smoke regularly. Identifying this susceptible subset of the population before the onset of nicotine dependence may encourage targeted early interventions to prevent regular smoking and/or minimize severity. While prospective neuroimaging in human populations can be challenging, preclinical neuroimaging models before chronic nicotine administration can help to develop translational biomarkers of disease risk. Chronic, intermittent nicotine $(0,1.2$, or 4.8 $\mathrm{mg} / \mathrm{kg} / \mathrm{d} ; N=10-11 /$ group) was administered to male Sprague Dawley rats for $14 \mathrm{~d}$; dependence severity was quantified using precipitated withdrawal behaviors collected before, during, and following forced nicotine abstinence. Resting-state fMRI functional connectivity (FC) before drug administration was subjected to a graph theory analytical framework to form a predictive model of subsequent individual differences in nicotine dependence. Whole-brain modularity analysis identified five modules in the rat brain. A metric of intermodule connectivity, participation coefficient, of an identified insular-frontal cortical module predicted subsequent dependence severity, independent of nicotine dose. To better spatially isolate this effect, this module was subjected to a secondary exploratory modularity analysis, which segregated it into three submodules (frontal-motor, insular, and sensory). Higher FC among these three submodules and three of the five originally identified modules (striatal, frontal-executive, and sensory association) also predicted dependence severity. These data suggest that predispositional, intrinsic differences in circuit strength between insular-frontal-based brain networks before drug exposure may identify those at highest risk for the development of nicotine dependence.

Significance Statement

Developing biomarkers of individuals at high risk for addiction before the onset of this brain-based disease is essential for prevention, early intervention, and/or subsequent treatment decisions. Using a rodent model of nicotine dependence and a novel data-driven, network-based analysis of resting-state fMRI data collected before drug exposure, functional connections centered on an intrinsic insular-frontal module predicted the severity of nicotine dependence after drug exposure. The predictive capacity of baseline network measures was specific to inter-regional but not within-region connectivity. While insular and frontal regions have consistently been implicated in nicotine dependence, this is the first study to reveal that innate, individual differences in their circuit strength have the predictive capacity to identify those at greatest risk for and resilience to drug dependence.

\section{Introduction}

Approximately $60 \%$ of the US population report ever smoking cigarettes (Surgeon General, 2014), yet only 16\% of the US pop-

Received Jan. 10, 2019; revised March 27, 2019; accepted April 6, 2019.

Author contributions: E.A.S. designed research; L.-M.H., J.K.B., and H.L. performed research; L.-M.H. and X.L. contributed unpublished reagents/analytic tools; L.-M.H. and X.L. analyzed data; L.-M.H., R.J.K., Y.Y., and E.A.S. wrote the paper.

*L-M.H. and R.J.K. contributed equally to this work.

The authors declare no competing financial interests.

This work was supported by the Intramural Research Program of National Institute on Drug Abuse/National Institutes of Health, Food and Drug Administration Center on Tobacco Products (Grant NDA 13001-001-00000 to E.A.S.) and a postdoctoral fellowship award from the Canadian Institutes of Health Research to R.J.K. (FRN 152478). The authors declare no competing financial interests.

Correspondence should be addressed to Elliot A. Stein at estein@nih.gov. ulation are regular smokers (Centers for Disease Control and Prevention, 2018). Why only a subset of individuals transition from early smoking experimentation to nicotine dependence remains unknown. Genetic polymorphisms at the $15 \mathrm{q}$ nicotinic acetylcholine receptor ( $\mathrm{nAChR}$ ) gene cluster have been consistently associated with increased risk of smoking (Saccone et al., 2007; Bierut et al., 2008; Sherva et al., 2008; Liu et al., 2019). Moreover, using resting-state (rs) fMRI functional connectivity

L.-M. Hsu's present address: Department of Radiology and BRIC, University of North Carolina at Chapel Hill, 130 Mason Farm Road, Chapel Hill, NC 27599.

J. K. Brynildsen's present address: Department of Systems Pharmacology and Translational Therapeutics, Perelman School of Medicine, University of Pennsylvania, 3400 Civic Center Boulevard, Philadelphia, PA 19104.

https://doi.org/10.1523/JNEUROSCI.0140-19.2019

Copyright $\odot 2019$ the authors 
(FC), we previously identified a brain circuit-based biomarker of smoking dependence severity modulated by this genetic polymorphism (Hong et al., 2009, 2010). However, whether this and/or additional circuits change during the development of nicotine addiction or whether they differ before smoking initiation and thus predispose individuals to subsequent development of nicotine dependence remains unknown. To date, beyond genetic risk factors, no clinically useful biomarkers of dependence risk have been definitively identified for nicotine or any other drug of abuse. Early risk detection could provide a crucial window for intervention before the development of nicotine dependence.

Noninvasive neuroimaging offers multiple advantages as a potential biomarker: it provides a sensitive measure of brain function in health and disease; is easily implemented in prospective, retrospective, and longitudinal experimental designs; and, crucially, is translatable between clinical populations and preclinical disease models since the same signal can be recorded from anatomically and functionally homologous circuits and networks (Lu et al., 2012). Thus, many of the limitations of prospective and longitudinal studies in human populations, including subject recruitment, retention, and psychiatric comorbidity, are obviated in preclinical imaging studies.

Graph theory-based analyses suggest that the brain is organized into and functions as a complex network of anatomically connected and functionally interacting brain regions or nodes (Bullmore and Sporns, 2009) with a "modular" architecture supporting segregation and integration of communication between nodes (Sporns, 2013). In this framework, a module in a network is defined as a set of nodes that is densely connected within the module and sparsely connected to nodes outside of the module (Newman, 2006). Summary measures of network modules can characterize different aspects of local and global connectivity, which can provide a network metric that may be applied to compare cohorts. For example, intermodule connectivity, characterized by participation coefficient (PC), indicates the level of interactions with nodes of other modules, while intramodule connectivity, characterized by within-module degree (WD), quantifies the level of connectivity of nodes within a module. A handful of studies have addressed network organization of the rodent brain (Liang et al., 2018), which, like humans, can vary with disease (Ash et al., 2016). Brain modules (He et al., 2009) and the interactions within and between modules (Liang et al., 2015) differ in substance use disorder. However, no study to date has investigated whether baseline network organization of the rat brain can quantitatively predict any disease outcome, let alone dependence following drug exposure.

To this end, we used a whole-brain, data-driven approach, with no a priori regional brain assumptions, and focused on baseline intramodule and intermodule connectivity, WD and PC, respectively, as dependent variable predictive features of subsequent nicotine dependence, which we induced based on a previous rodent nicotine delivery method in rodents (Brynildsen et al., 2016). We chose one aspect of the rodent nicotine withdrawal syndrome, somatic behavioral signs, as a proxy for assessing nicotine dependence. Such somatic signs can be observed spontaneously following forced abstinence or can be acutely precipitated following the administration of the nonspecific nAChR antagonist mecamylamine. As the intensity of the observed behavioral signs scales with the dose and duration of the administered nicotine (Malin et al., 1992), and as withdrawal severity in humans can predict smoking relapse (Piasecki et al., 1998, 2000), we used a composite score based on the number and frequency of somatic
A

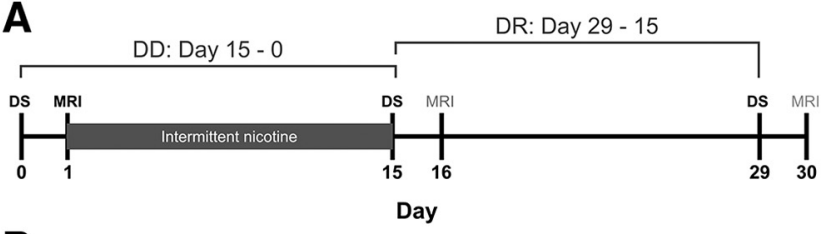

B
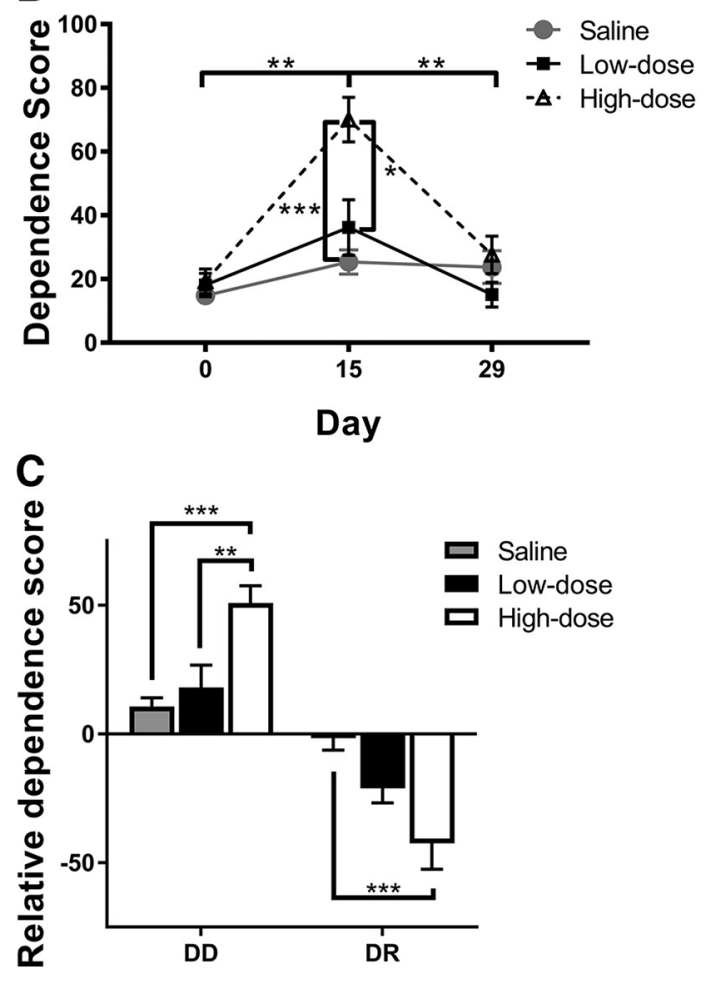

Figure 1. $A$, Timeline of experimental procedures demonstrating MRI and dependence score (DS) assessment. $\boldsymbol{B}$, Composite somatic withdrawal scores following a mecamylamine (1.5 $\mathrm{mg} / \mathrm{kg}$ ) challenge measured from groups of rats receiving 2 weeks of saline, low-dose nicotine $(1.2 \mathrm{mg} / \mathrm{kg} / \mathrm{d})$, or high-dose nicotine $(4.8 \mathrm{mg} / \mathrm{kg} / \mathrm{d})$. Rats that received high-dose nicotine showed significantly greater dependence score on day 15 than the saline $(p<0.001)$ and low-dose nicotine $(p<0.05)$ groups. The dependence level in high-dose nicotine treated rats on day 15 was significantly reduced on day 29 , returning to predrug baseline levels (day 0 ). C, Relative dependence scores for DD (days 15-0) and DR (days 29-15) for all groups. High-dose nicotine treatment elevated DD relative to saline $(p<0.001)$ and low-dose nicotine treatment $(p<0.01)$ and decreased DR relative to saline treatment $(p<0.001)$. Data are presented as the mean \pm SEM. All $p$ values are corrected for multiple comparisons $\left({ }^{*} p<0.05,{ }^{* *} p<0.01\right.$, $\left.{ }^{* * *} p<0.001\right)$.

withdrawal behaviors as the independent variable in our brain network analyses to parallel human dependence severity.

\section{Materials and Methods}

Subjects and general experimental design. Groups of male Sprague Dawley rats $(275-300 \mathrm{~g})$ were administered saline $(N=11)$, low-dose nicotine $(1.2 \mathrm{mg} / \mathrm{kg} / \mathrm{d} ; N=11)$, or high-dose nicotine $(4.8 \mathrm{mg} / \mathrm{kg} / \mathrm{d} ; N=10)$ for $14 \mathrm{~d}$ using an implanted osmotic minipump. All rats underwent MRI scanning on the day before pump implantation [day 1 (baseline)]. Assessments of somatic signs of nicotine withdrawal occurred one day before the MRI scan [i.e., day 0 (baseline)], and dependence development was assessed on day 15 (peak dependence) and day 29 (extended abstinence). Additional MRI scans were performed on day 16 following $14 \mathrm{~d}$ of nicotine administration and day 30 following $14 \mathrm{~d}$ of abstinence. As the goal of this analysis was to develop a biomarker of risk/resilience, only imaging data collected before drug administration (day 1) were used to predict subsequent nicotine dependence severity (Fig. 1A, experimental timeline). All study procedures were approved by the Animal Care and 
Table 1. List of anatomical ROIs and abbreviations

\begin{tabular}{|c|c|c|c|c|c|}
\hline Region & & & Region & & Region \\
\hline Cortex & & TeA & Temporal association cortex & Thalamus & \\
\hline FrA & Frontal association cortex & M0 & Medial orbital cortex & $\mathrm{AM}$ & Anteromedial thalamic nucleus \\
\hline $\mathrm{Fr} 3$ & Frontal cortex, area 3 & V0 & Ventral orbital cortex & PT & Paratenial thalamic nucleus \\
\hline M1 & Primary motor cortex & LO & Lateral orbital cortex & Rt & Reticular thalamic nucleus \\
\hline M2 & Secondary motor cortex & DLO & Dorsolateral orbital cortex & VL & Ventrolateral thalamic nucleus \\
\hline $\operatorname{PrL}$ & Prelimbic cortex & RSD & Retrosplenial dysgranular cortex & VA & Ventral anterior thalamic nucleus \\
\hline IL & Infralimbic cortex & RSGC & Retrospenial granular cortex, c region & VPL & Ventral posterolateral thalamic nucleus \\
\hline AIV & Agranular insular cortex, ventral part & LEnt & Lateral entorhinal cortex & VPM & Ventral posteromedial thalamic nucleus \\
\hline AID & Agranular insular cortex, dorsal part & PRh & Perirhinal cortex & LDDM & Laterodorsal thalamic nucleus, dorsomedial \\
\hline $\mathrm{DI}$ & Dysgranular insular cortex & Ect & Ectorhinal cortex & LDVL & Laterodorsal thalamic nucleus, ventrolateral \\
\hline $\mathrm{Gl}$ & Granular insular cortex & AuV & Secondary auditory cortex, ventral area & AVDM & Anteroventral thalamic nucleus, dorsomedial part \\
\hline AIP & Agranular insular cortex, posterior part & Au1 & Primary auditory cortex & AVVL & Anteroventral thalamic nucleus, ventrolateral part \\
\hline $\mathrm{Cg} 1$ & Cingular cortex, area 1 & $\mathrm{AuD}$ & Secondary auditory cortex, dorsal area & MDM & Mediodorsal thalamic nucleus, medial part \\
\hline $\mathrm{Cg} 2$ & Cingulate cortex, area 2 & LPtA & Lateral parietal association cortex & MDL & Mediodorsal thalamic nucleus, lateral part \\
\hline S1 & Primary somatosensory cortex & MPtA & Medial parietal association cortex & $\mathrm{Rh}$ & Rhomboid thalamic nucleus \\
\hline S1J & Primary somatosensory cortex, jaw region & PtPR & Parietal cortex, posterior area, rostral part & $\operatorname{Re}$ & Reuniens thalamic nucleus \\
\hline S1ULp & Primary somatosensory cortex, upper lip region & PtPD & Parietal cortex, posterior area, dorsal part & LPMR & Lateral posterior thalamic nucleus, mediorostral part \\
\hline S1DZ0 & Primary somatosensory cortex, oral dysgranular zone & Hippocampus & & LPMC & Lateral posterior thalamic nucleus, mediocaudal part \\
\hline S1DZ & Primary somatosensory cortex, dysgranular zone & dHIPP & Dorsal hippocampus & LPLR & Lateral posterior thalamic nucleus, laterorostral part \\
\hline S1FL & Primary somatosensory cortex, forelimb region & vHIPP & Ventral hippocampus & LPLC & Lateral posterior thalamic nucleus, laterocaudal part \\
\hline $\mathrm{S} 1 \mathrm{BF}$ & Primary somatosensory cortex, barrel field & Striatum & & Po & Posterior thalamic nuclear group \\
\hline S1HL & Primary somatosensory cortex, hindlimb region & $\mathrm{NcbC}$ & Nucleus accumbens core & $\mathrm{PF}$ & Parafascicular thalamic nucleus \\
\hline S1Tr & Primary somatosensory cortex, trunk region & NcbS & Nucleus accumbens shell & V1 & Primary visual cortex \\
\hline S2 & Secondary somatosensory cortex & $\mathrm{CPu}$ & Caudate putamen & V2ML & Secondary visual cortex, mediolateral area \\
\hline DP & Dorsal peduncular cortex & & & V2MM & Secondary visual cortex, mediomedial area \\
\hline
\end{tabular}

Use Committee of the National Institute on Drug Abuse, Intramural Research Program.

Intermittent nicotine administration. An intermittent, passive nicotine administration approach, developed by our group (Brynildsen et al., 2016), used a modified osmotic minipump (model 2ML4, Alzet) in combination with a Lynch coil (PE60 tubing) to deliver intraperitoneal saline or nicotine (nicotine hydrogen tartrate salt, dissolved in $0.9 \%$ sodium chloride and $\mathrm{pH}$ adjusted to $7.2 \pm 0.5$ ) in a fixed interval $1 \mathrm{~h}-\mathrm{ON}-1$ h-OFF pattern for $\sim 14 \mathrm{~d}$, starting on day 1 . This method of intermittent nicotine delivery produces consistent and precise phasic delivery of nicotine and induces heightened and persistent dose-dependent precipitated withdrawal behaviors compared with continuous minipump delivery. Doses of 1.2 and $4.8 \mathrm{mg} / \mathrm{kg} / \mathrm{d}$ were chosen based upon previous dose-dependent studies of nicotine dependence (Malin et al., 1992; Benwell et al., 1995; Watkins et al., 2000; Vann et al., 2006). Pumps were implanted under isoflurane (2-3\%) anesthesia.

Behavioral assessment of nicotine dependence. Rats were placed into a square Plexiglas chamber $(35 \times 35 \times 40 \mathrm{~cm})$ on days 0,15 , and 29 and were allowed to habituate for $10 \mathrm{~min}$. Precipitated withdrawal was induced with an acute injection of mecamylamine $\mathrm{HCl}(1.5 \mathrm{mg} / \mathrm{kg}$, s.c.; Sigma-Aldrich). Mecamylamine, a nonselective, noncompetitive nicotinic acetylcholine receptor antagonist, acutely produces a suite of somatic and affective withdrawal behavioral signs in nicotine-exposed rats (Malin et al., 1992), similar to those observed following naloxone administration in opioid-dependent rats (Koob et al., 1992). Mecamylamine-precipitated somatic and affective withdrawal signs increase in a dose-dependent manner with nicotine exposure (EppingJordan et al., 1998; Rezvani and Levin, 2001; Damaj et al., 2003). The frequency of somatic withdrawal behaviors (ptosis, teeth chattering, chewing, gasping, writhing, head shakes, body shakes, tremors, blinks, yawns, seminal ejaculation, genital licks, hindfoot scratches, and escape attempts) were summed over a 50 min observation period to generate a composite "dependence score." Relative dependence scores were calculated as the difference in the dependence score between days, denoted as "dependence development" [DD; i.e., the difference between the dependence score observed on day 15 (peak dependence) and that recorded on day 0 (baseline)] and "dependence reversal" [DR; i.e., the difference between the dependence score on day 29 (after $14 \mathrm{~d}$ of abstinence) and that observed on day 15 (peak dependence)].

fMRI animal preparation and data acquisition. Imaging was performed using a Bruker Biospin $9.4 \mathrm{~T} / 30 \mathrm{~cm}$ scanner. Rats were anesthetized using low-dose isoflurane $(0.25-0.5 \%)$ and dexmedetomidine $(0.015 \mathrm{mg} / \mathrm{kg} / \mathrm{h}$, s.c.) and were placed in a customized MRI cradle for head fixation. BOLD acquisition occurred at least $1 \mathrm{~h}$ after anesthesia induction to ensure that monitored physiology (heart rate, blood pressure, respiration, and body temperature) was stable (Lu et al., 2012; Brynildsen et al., 2017).

A RARE (rapid imaging with refocused echoes) sequence was used to acquire high-resolution, T2-weighted anatomical images, which were used for regional anatomical localization. A gradient-echo EPI sequence (FOV, $32 \mathrm{~mm}$; matrix size, $64 \times 64$; repetition time, $1 \mathrm{~s}$; echo time, $15 \mathrm{~ms}$; 15 slices; $1 \mathrm{~mm}$ thickness) was used to acquire a BOLD fMRI dataset of 900 volumes.

FC and modularity analysis. Using AFNI (Cox, 1996), preprocessing steps included discarding the first 10 volumes to allow the MRI signal to reach steady state, slice timing correction, motion correction, and spatial smoothing ( $0.6 \mathrm{~mm}$ full-width at half-maximum). The fMRI images were aligned to their corresponding T2-weighted images and then normalized to a common 3D space aligned with a rat stereotaxic atlas (Lu et al., 2010). To improve the accuracy of spatial normalization, fMRI data from each rat were aligned to the group-averaged images. Finally, the normalized images were linearly detrended, bandpass filtered $(0.01<\mathrm{f}<0.5 \mathrm{~Hz})$, and the six head movement parameters were regressed from the signal.

We spatially defined 118 ROIs based on a stereotaxic atlas (Paxinos and Watson, 2007), which included 44 bilateral cortical and 15 subcortical regions (Table 1, list of ROIs). To conduct graph theory-based analyses, we constructed a whole-brain network, using ROIs as nodes and FC between ROIs as edges. For each rat, we extracted the time course from each of the ROIs and computed the Pearson correlation between every pair of nodes to form a $118 \times 118 \mathrm{rsFC}$ community structure matrix. Correlation coefficients were converted to Fisher's $z$ values before applying algebraic and statistical operations. Next, rsFC matrices were averaged across all rats to produce a mean rsFC matrix. To decompose the lowest hierarchy components in a defined network, the matrix was thresholded at $5 \%$ edge density $(r=0.25)$ to keep the strongest connections while ensuring that at least $95 \%$ of the network nodes were connected (Power et al., 2011, 2013; Hsu et al., 2016). The Newman (2006) spectral algorithm was used for modular detection. We observed high similarity, measured by normalized mutual information (MIn), between our results, using a threshold of $5 \%$ edge density, and results thresholded at $10 \%(\mathrm{MIn}=0.84), 15 \%(\mathrm{MIn}=0.77)$, and $20 \%$ edge density $(\mathrm{MIn}=0.79)$. Two standard graph theory network metrics, 
PC and WD, were used to assess intermodule and intramodule interactions and connectivity.

The modularity index $Q$, quantifies the efficacy of partitioning a network into modules by evaluating the difference between the actual number of intramodule connections and the expected number for the same modules in a randomized network (Newman, 2004). The objective of a modular detection procedure is to find a specific partition that maximizes $Q$. To examine whether a real modular network was significantly different from random graphs, we randomized the original network with preserved strength distribution 1000 times and calculated the mean $(\mu)$ and SD $(\sigma)$ of those modularity values (Maslov and Sneppen, 2002). We compared the modularity $Q$ of the real network to those values, which measures how many SDs the real modularity is above the mean for the random graph, as follows:

$$
z=\frac{Q-\mu}{\sigma}
$$

Modular assignment provides the basis for assessing the patterns of intramodule and intermodule connectivity (Guimerà and Nunes Amaral, 2005). A standard network metric, PC, was used to assess intermodule interactions and connectivity and was calculated as follows:

$$
P C_{i}=1-\sum_{s=1}^{N_{M}}\left(\frac{k_{i s}}{k_{i}}\right)^{2},
$$

where $N_{\mathrm{M}}$ is the number of modules, $k_{\mathrm{is}}$ is the averaged connection between module $i$ and module $s$, and $k_{\mathrm{is}} / k_{\mathrm{i}}$ represents the ratio of connections within a module. If the connections of a module are entirely restricted to itself, $k_{\text {is }} / k_{\mathrm{i}}$ has a value of 1 and $\mathrm{PC}$ has a value of 0 . If the connections of a module are evenly distributed among all other modules, PC will be maximal, approaching a value of 1 .

To investigate how nodes connect to other nodes within a module, the WD $z$ score was used to assess intramodule interaction and was calculated as follows:

$$
Z_{i}=\frac{k_{i}-\bar{k}_{s_{i}}}{\sigma_{k_{s i}}},
$$

where $k_{\mathrm{i}}$ is the number of links of node $i$ to other nodes in module $s_{\mathrm{i}}, \bar{k}_{\mathrm{si}}$ is the average of $k$ over all the nodes in $s_{\mathrm{i}}$, and $\sigma_{\mathrm{ksi}}$ is the SD of $k$ in $s_{\mathrm{i}}$.

Experimental design and statistical analyses. A generalized linear mixed-effects (LME) model in MATLAB (MathWorks) was used to model the relative dependence scores (DD and DR), including fixed effects of nicotine dose and experimental day and their interaction. A second generalized LME design modeled the effect of network metrics ( $\mathrm{PC}$ and $\mathrm{WD}$ ) and nicotine dose on dependence in the exposed nicotine rats (low- and high-dose nicotine). As a control, a separate LME model examined the effect of network metrics on the measure of nicotine dependence in saline-treated rats. To assess whether network PC and WD at baseline (day 1) predicted dose-dependent nicotine dependence following nicotine administration (day 15) and following withdrawal (day 29), only day 1 imaging data were used in the model.

We identified an insular-frontal module (see Results) that contributed to the relationship between DD/DR and between-module FC. To determine which regions within this module were driving the relationship, a second, exploratory modularity analysis was conducted within this insular-frontal module. A stepwise linear regression was conducted to evaluate the significant contributions of each derived submodule to the correlation between the insular-frontal module rsFC with other modules and DD or DR. As a post hoc analysis, we computed the between-module connectivity for each rat by averaging functional correlations across all pairs of anatomical ROIs between the identified insular-frontal module and all other modules identified by our predictive measure. Finally, the FC between modules was correlated with DD/DR. For all analyses and all post hoc comparisons, a Bonferroni-corrected $p$ value of $<0.05$ was considered significant. The significance for the PC analysis was corrected for five modules and two groups (nicotine and saline treatment), while the
WD significance analysis was corrected for the number of nodes within its corresponding module.

\section{Results}

Nicotine administration dose-dependently increases precipitated withdrawal dependence score

There was a significant main effect of dose $\left(F_{(2,29)}=11.42, p<\right.$ $0.001)$ and a significant dose $\times$ day $\left(F_{(4,45)}=8.4, p<0.001\right.$; Fig. $1 B)$ interaction for the mecamylamine-induced composite dependence score. Simple effects contrasts indicated that the high-dose nicotine group was significantly more dependent compared with the saline $\left(p_{\text {corrected }}<0.001\right)$ and low-dose nicotine $\left(p_{\text {corrected }}<0.05\right)$ groups on day 15 . Moreover, within the high-dose group, there was a significant main effect of day $\left(F_{(2,27)}=22.7, p_{\text {corrected }}<0.001\right)$, with rats showing significantly greater dependence on day $15 \mathrm{com}-$ pared with either baseline (day $0, p_{\text {corrected }}<0.01$ ) or following extended abstinence (day 29; $p_{\text {corrected }}<0.01$ ).

Similar results were observed for relative behavioral scores, DD (days 15-0) and DR (days 29-15). There was a significant main effect of dose for DD $\left(F_{(2,29)}=3.386, p<0.05\right)$ and DR $\left(F_{(2,29)}=3.437, p<0.05\right.$; Fig. $\left.1 C\right)$. High-dose nicotine increased the relative dependence score for DD compared with saline $(p<$ $0.001)$ and low-dose nicotine $(p<0.01)$ groups and decreased the relative dependence score for DR relative to the saline group $(p<0.001)$.

Thus, using a validated behavioral technique to assess nicotine dependence severity (Malin et al., 1992; Malin, 2001) in combination with a previously described animal model of nicotine dependence (Brynildsen et al., 2016), we observed nicotine dosedependent increases in dependence severity.

\section{Intermodule but not intramodule connectivity predicts dependence severity}

Modularity analyses identified five brain modules named, based upon their major anatomical constituents and associated function, as follows: insular-frontal, thalamic, frontal-executive, sensory association, and striatal (Fig. 2A,B). Anatomical ROIs localized within each module are listed in Table 2.

Intermodule (PC; Fig. 2C) and intramodule (WD) interactions before drug administration were entered into the model to predict the severity of subsequent nicotine dependence (DD) and the remission of dependence (DR) following sustained abstinence. Of the five identified modules, only the PC of the insularfrontal module (Fig. 3A) in nicotine-treated rats at baseline correlated significantly with $\mathrm{DD}\left(F_{(1,10)}=15.5, p_{\text {corrected }}<0.05\right.$; Fig. $3 B)$ and $\operatorname{DR}\left(F_{(1,10)}=7.1, p_{\text {corrected }}<0.05\right.$; Fig. $\left.3 C\right)$ and did so independent of nicotine dose. No significant association was observed with DD and DR in saline control rats. We compared the correlation coefficients of nicotine-exposed and saline-exposed rats. The association of DD and PC of the insular-frontal module for nicotine exposed rats $(r=0.70,95 \% \mathrm{CI}=0.38-0.86)$ was significantly stronger $(p<0.05)$ compared with saline control rats $(r=-0.01, p=0.97,95 \% \mathrm{CI}=-0.60$ to 0.59$)$, and the association of DR and PC of the insular-frontal module in nicotine-exposed rats $(r=-0.56,95 \% \mathrm{CI}=-0.80$ to -0.17$)$ was significantly stronger $(p<0.05)$ compared with saline control rats $(r=0.31, p=0.35,95 \% \mathrm{CI}=-0.36$ to -0.77$)$. Thus, communication strength between this insular-frontal module and all other identified modules before drug exposure predicted dependence severity and its abstinence-induced reversal. There was no significant relationship between any module PC and dependence when comparing baseline with extended abstinence (days 29-0), suggesting that the development of and recovery 
A

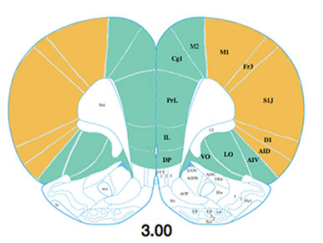

3.00

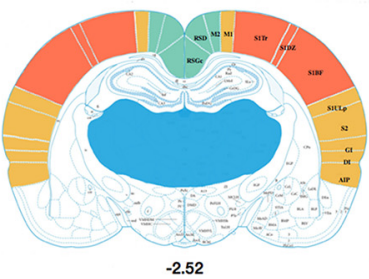

Insular-frontal module

Frontal executive module

Sensory association module
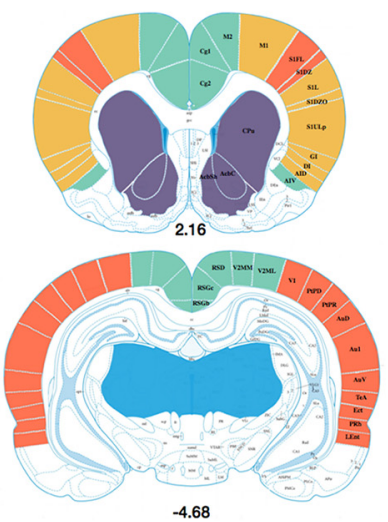

Striatal module Thalamic module
B

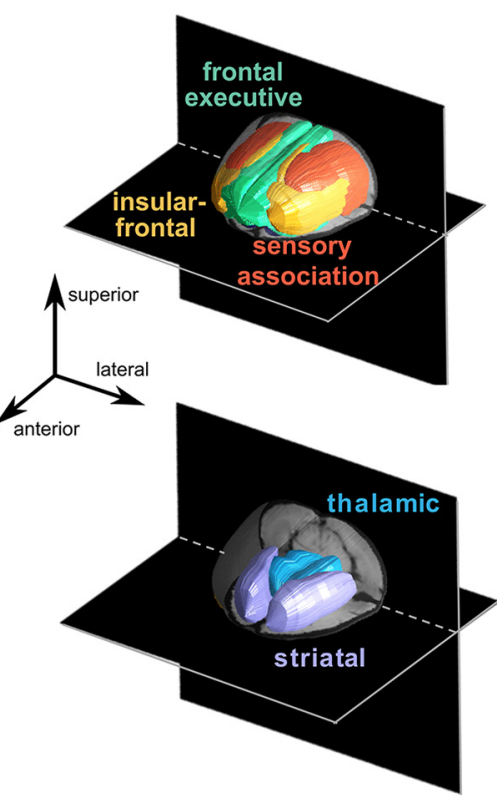

C

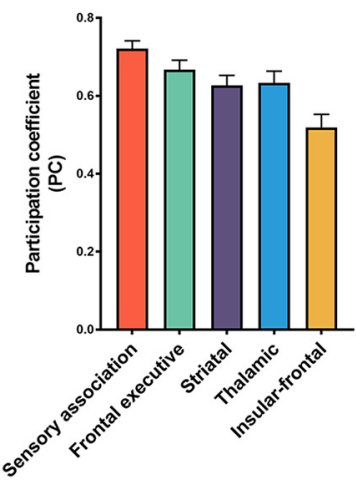

Figure 2. $\quad \boldsymbol{A}$, Brain regions $(N=118$ ) selected as input nodes in the modularity analysis color coded according to their subsequent modular affiliation (shown in $\boldsymbol{B})$ and represented as an underlay on a stereotaxic brain atlas (distance from bregma in millimeters is given below each slice). $\boldsymbol{B}$, Results from the modularity analysis demonstrating the five functional networks identified and illustrated in 3D color-coded composite images. C, Mean PC of each module.

Table 2. Anatomical constituents of the five identified brain modules

\begin{tabular}{|c|c|c|}
\hline Module & Brain regions & Abbreviations \\
\hline \multirow[t]{5}{*}{ Insular-frontal } & Frontal cortex & $\mathrm{Fr} 3$ \\
\hline & Insular cortex & $D I, G I, A I P$, and AID \\
\hline & Orbital cortex & DLO \\
\hline & Primary motor cortex & M1 \\
\hline & Somatosensory cortex & S1J, S1UpL, S1DZO, and S2 \\
\hline \multirow[t]{8}{*}{ Frontal executive } & Cingulate cortex & $\mathrm{Cg} 1$ and $\mathrm{Cg} 2$ \\
\hline & Frontal association cortex & FrA \\
\hline & Insular cortex & AIV \\
\hline & Limbic cortex & PrL and IL \\
\hline & Orbital cortex & $\mathrm{MO}, \mathrm{V} 0$, and LO \\
\hline & Retrosplenial cortex & RSD and RSGC \\
\hline & Secondary motor cortex & M2 \\
\hline & Secondary visual cortex & V2MM and right-V2ML \\
\hline \multirow[t]{6}{*}{ Sensory association } & Auditory cortex & AuV, Au1, and AuD \\
\hline & Parietal cortex & LPtA, MPtA, PtPR, and PtPD \\
\hline & Primary somatosensory cortex & S1, S1DZ, S1FL, S1BF, S1HL, and S1Tr \\
\hline & Rhinal cortex & Lent, PRh, and Ect \\
\hline & Temporal association cortex & TeA \\
\hline & Visual cortex & V1 and left-V2ML \\
\hline \multirow[t]{2}{*}{ Striatal } & Caudate putamen & CPu \\
\hline & Nucleus accumbens (core and shell) & $\mathrm{NcbC}$ and $\mathrm{NcbS}$ \\
\hline \multirow[t]{9}{*}{ Thalamic } & Anteromedial thalamic nucleus & AM \\
\hline & Lateral posterior thalamic nucleus & LPMR, LPMC, LPLR, and LPLC \\
\hline & $\begin{array}{l}\text { Laterodorsal and anteroventral } \\
\text { thalamic nuclei }\end{array}$ & LDMM, LDVL, AVDM, and AVVL \\
\hline & Mediodorsal thalamic nucleus & MDM and MDL \\
\hline & Parafascicular thalamic nucleus & PF \\
\hline & Paratenial thalamic nucleus & PT \\
\hline & Posterior thalamic nuclear group & Po \\
\hline & Rhomboid and reuniens thalamic nuclei & Rh and Re \\
\hline & $\begin{array}{l}\text { Ventral thalamic } \\
\text { nucleus }\end{array}$ & $V L, V A, V P L$, and VPM \\
\hline
\end{tabular}

Abbreviations are based on those found in Paxinos and Watson (2007).

from nicotine dependence were linked to insular-frontal module function.

In contrast, there were no significant associations between $\mathrm{WD}$ and dependence for any node (all $p$ values $>0.05$ ), suggesting that within-module connectivity does not index future drug use, at least in this model. Thus, WD was not included in subsequent analyses.

The insular-frontal module parcellates into three dependence predictive submodules

Since the insular-frontal module comprised anatomically heterogeneous structures, we sought to delineate which regions within this module contributed to the observed intermodule brain-behavior relationship. A secondary exploratory modularity analysis, performed only upon the insular-frontal module, identified three submodules (Fig. 3D) termed insular (composed of DI, GI, AIP, and AID), frontal-motor (DLO, Fr3, and M1), and sensory (S1J, S1UpL, S1DZO, and S2).

We next performed a stepwise linear regression to determine the contribution of each of the three submodules to the prediction of nicotine dependence severity (Fig. $3 E-I)$. PC of the insular $\left(p_{\text {corrected }}<0.001\right.$; Fig. $\left.3 E\right)$, frontal-motor $\left(p_{\text {corrected }}<0.01\right.$; Fig. $3 F$ ), and sensory ( $p_{\text {corrected }}<0.05$; Fig. $3 G$ ) submodules all predicted DD, while PC of the insular $\left(p_{\text {corrected }}<0.05\right.$; Fig. $\left.3 H\right)$ and frontal-motor $\left(p_{\text {corrected }}<0.01\right.$; Fig. $\left.3 I\right)$ submodules predicted DR. Thus, intermodule connectivity of all three insular-related submodules predicted dependence development, whereas intermodule connectivity of only the insular and frontal-motor submodules predicted dependence reversal following 2 weeks of abstinence.

FC between insular-frontal submodules and the remaining modules predicts DD and DR

We next interrogated the circuit mechanisms underlying the identified insular-based modular (and submodular) predictors of DD. A post hoc stepwise linear regression of the connectivity from each of the three predictive insular-frontal submodules with the four remaining initially identified modules (i.e., striatal, thalamic, frontal-executive, and sensory association; Fig. 4) found that rsFC between the insular submodule and both the striatal $\left(p_{\text {corrected }}<0.05 ;\right.$ Fig. $\left.4 B\right)$ and frontal-executive ( $p_{\text {corrected }}$ $<0.05$; Fig. $4 C$ ) modules predicted DD, accounting for, re- 

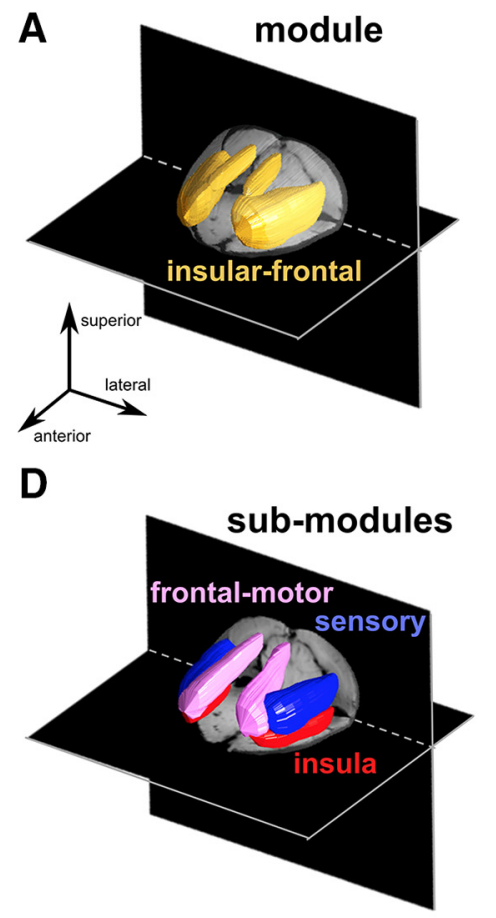

B

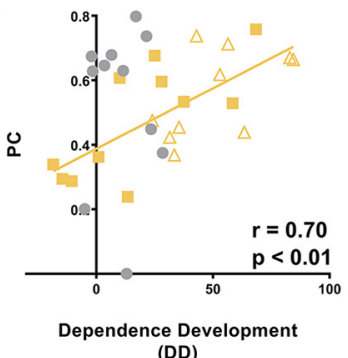

(DD)

E

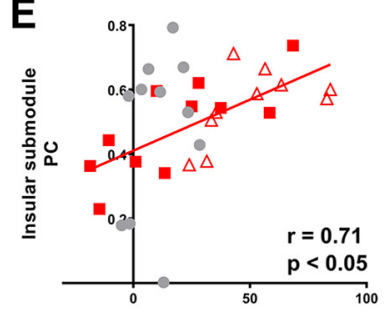

Dependence Development (DD)

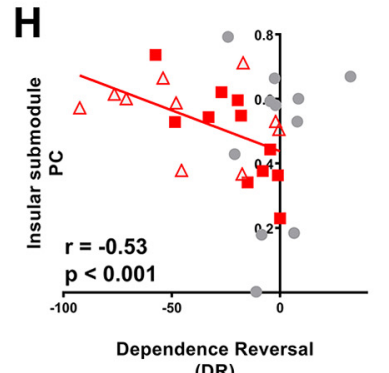

(DR)
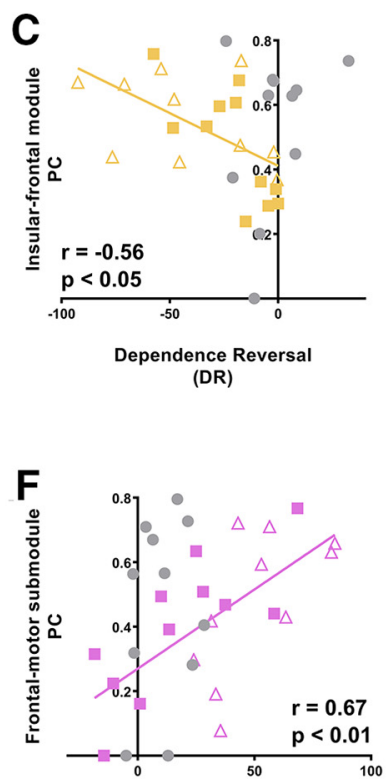

Dependence Development (DD)

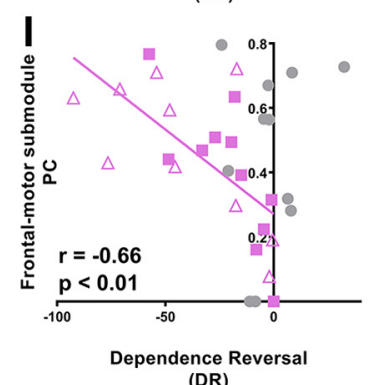

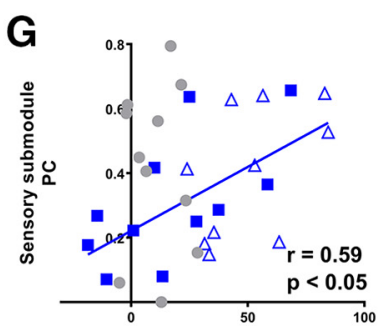

Dependence Development (DD) $\square$ Low dose
$\triangle$ High dose

Figure 3. $\boldsymbol{A}-\boldsymbol{C}$, The $P C$ of the insular-frontal module $(\boldsymbol{A})$ significantly correlated with changes in $D D(\boldsymbol{B})$ and $D R(\boldsymbol{C})$. $\boldsymbol{D}, A$ secondary modularity analysis limited to the identified insular-frontal module revealed three color-coded submodules illustrated in the 3D brain rendering. $\boldsymbol{E}-\boldsymbol{G}$, Using the three submodules, DD was significantly predicted by PC of insular $(\boldsymbol{E})$, frontal-motor $(\boldsymbol{F})$, and sensory $(\boldsymbol{G})$ submodules. $\boldsymbol{H}, \boldsymbol{I}$, DR was significantly predicted by PC of insular $(\boldsymbol{H})$ and frontal-motor $(\boldsymbol{I})$ submodules. Note that data from saline rats (in gray) are graphically represented but not included in the regression analyses. All $p$ values were Bonferroni corrected.

spectively, $37.9 \%$ and $31.2 \%$ of the variance in the model. Additionally, FC between the frontal-motor submodule and the sensory association module also predicted DD $\left(p_{\text {corrected }}<\right.$ 0.01 ; Fig. $4 D$ ), accounting for $39.6 \%$ of the model variance. Once again, despite the nicotine dose relationship observed in withdrawal behaviors, no dose relationship was found in our predictive network models.

After prolonged abstinence, DR was predicted by the FC between the insular submodule and the striatal module $\left(p_{\text {corrected }}<\right.$ 0.05 ; Fig. $4 E$ ) and between the frontal-motor submodule and the sensory association module ( $p_{\text {corrected }}<0.05$; Fig. $\left.4 F\right)$, accounting for $29.1 \%$ and $37.1 \%$ of the variance, respectively. No other significant correlations were observed, and again, no nicotine dose relationship was found. Thus, while baseline insular FC with a frontal-executive module before drug exposure mechanistically explained the development of subsequent dependence, baseline connectivity between the frontal-motor submodule and sensory association module and between the insular submodule and striatal module were associated with both dependence development and recovery following abstinence.

\section{Discussion}

We applied a data-driven graph theory modularity analysis of resting-state BOLD FC data and identified five principal modules in the drug-naive rat brain. In a rodent model of nicotine dependence using precipitated withdrawal as an index of dependence severity, intermodule connectivity (PC) of a module comprising insular-frontal brain regions before drug exposure was predictive of nicotine dependence severity. As expected, the severity of dependence increased with nicotine dose; however, the brain-based dependence prediction score did not.

To determine the neuroanatomical specificity that contributed to the predictive power of the insular-frontal module, a post hoc exploratory modularity analysis identified three submodules, all of which were predictive of dependence development. To understand the circuit specificity driving this prediction, post hoc analyses identified a functional circuit between the insular submodule and both the striatal and the frontal-executive modules along with a second circuit between the frontal-motor submodule and the sensory association module that predicted nicotine dependence severity. As imaging was performed before any drug administration, these findings support the hypothesis that intrinsic insular-frontal circuit components are predispositional biomarkers of nicotine dependence risk. Finally, recovery from nicotine dependence following forced abstinence was predicted by connectivity both between the insular submodule and the striatal module, and between the frontal- 


\section{Modules}

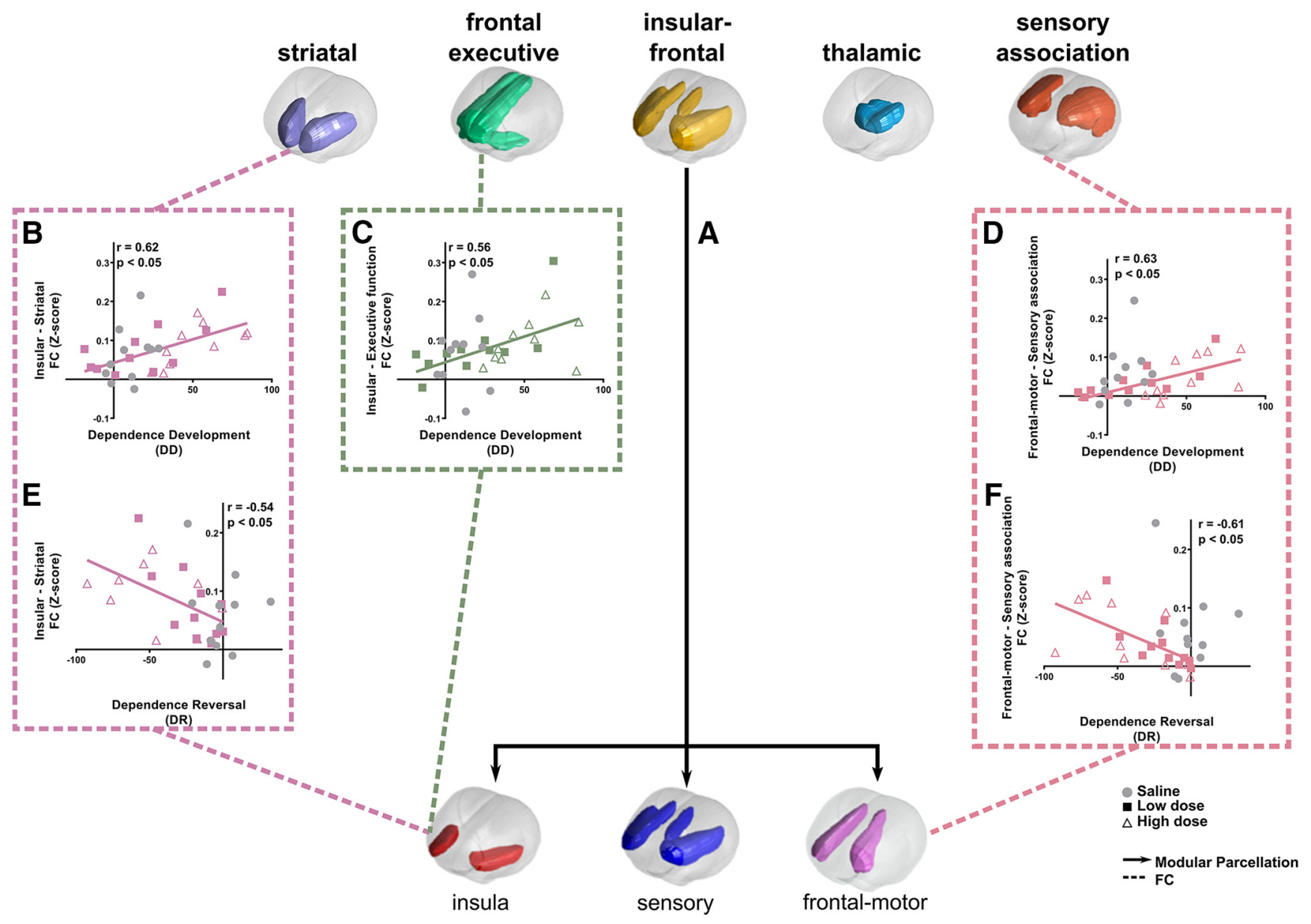

Submodules

Figure 4. A, Functional connectivity among the three submodules derived from the secondary modularity analysis of the insular-frontal module and all other modules. Solid arrowed lines depict the secondary modularity results segregating the insular-frontal module. Dashed lines represent $\mathrm{FC}$ between submodules and modules. $B, C, D D$ was significantly predicted by $\mathrm{FC}$ between the insular submodule and both striatal $(\boldsymbol{B})$ and frontal-executive modules $(\boldsymbol{C})$. D, Similarly, FC between the frontal-motor submodule and sensory association module predicted DD. $\boldsymbol{E}, \boldsymbol{F}, \mathrm{DR}$ was significantly predicted by $\mathrm{FC}$ between the insular submodule and striatal module $(\boldsymbol{E})$ and frontal-motor submodule and sensory association module $(\boldsymbol{F})$. Note that data from saline rats (in gray) are graphically represented but were not included in the regression analyses. All $p$ values were Bonferroni corrected.

motor submodule and the sensory association module. These effects were specific to intermodule connectivity $(\mathrm{PC})$ and were unrelated to intramodule connectivity (WD).

\section{Modularity analysis identified anatomically and functionally distinct brain regions}

Our modularity analysis instantiated cytoarchitectonically, anatomically, and functionally distinct brain regions. Notably, the striatal and thalamic modules closely followed known anatomy. While the other three modules (frontal-executive, insularfrontal, and sensory association) were less consistent with classical anatomical boundaries, they comprised nodes with shared functional properties. The frontal-executive module contained regions associated with executive control and decision making, including cingulate, prelimbic, infralimbic, and orbital frontal cortices (Kolb, 1984), and the sensory association module was composed of primary and secondary sensory and association areas, including the temporal association cortex (Vaudano et al., 1991). A previous report identified 7 modules in the rat brain
(Liang et al., 2018), whose anatomical makeup paralleled those observed here.

Individual variability in insular-frontal FC strength predicted nicotine dependence

Individual variability presumably accounts for the small fraction of ever-smokers who become regular smokers. Preclinical studies have also observed individual variability in the choice between drugs and other reinforcers (Ahmed et al., 2013) and behavioral measures linked to dependence severity (Belin et al., 2016), which themselves are associated with baseline differences in brain function (Tunstall and Kearns, 2015) and change with nicotine exposure (Palmatier et al., 2004). That our predictive networks were independent of nicotine dose, despite changes in behavioral signs of dependence severity with increasing nicotine dose suggests that brain-based traits have a predictive ability beyond purely pharmacological effects of nicotine.

The observed predictive brain networks were associated with increased insular and frontal connectivity, regions consistently 
implicated in nicotine dependence (Koob and Volkow, 2016). The insula is a highly interconnected, functionally heterogeneous cortical region that provides dynamic connectivity with cortical, limbic, and basal ganglia regions and is implicated in cognitive, emotional, and autonomic regulation (Craig, 2009). Homeostatically relevant interoceptive and exteroceptive environmental information are received, integrated, and processed within the insula to support contextually appropriate behavioral responding (Flynn, 1999; Kobayashi, 2011; Nomi et al., 2016). The computation of environmental salience is thought to occur within the so-called salience network (SN), which in humans consists primarily of the anterior insula and dorsal anterior cingulate cortex (Menon and Uddin, 2010). Despite the identification and definition of a rodent default-mode network (DMN; Lu et al., 2012) and its associated functional correlates (Hsu et al., 2016), the rat SN has yet to be well defined or functionally interrogated.

The insula contains a high density of nicotinic receptors (Perry et al., 2002; Picard et al., 2013), and its thickness changes with smoking (Zhang et al., 2011; Morales et al., 2014). Stroke damage to the insula disrupts cigarette smoking (Naqvi et al., 2007), and insular lesions in rodents disrupt compulsive behaviors (Belin-Rauscent et al., 2016). Insular rsFC to large-scale networks or their component regions is sensitive to nicotine withdrawal (Fedota et al., 2016; Sutherland et al., 2016). Abstinent smokers express enhanced circuit strength between the insula and the DMN (Fedota et al., 2018), which is thought to bias behavior toward endogenously generated stimuli (Sutherland et al., 2013). Individual variability in insular-frontal connectivity moderates the relationship between smoking and impulsivity (Clewett et al., 2014) and changes as a function of genetic susceptibility to drug use (Villafuerte et al., 2012).

While it remains unclear whether differences in insular connectivity in humans are due to nicotine dependence or preexisting susceptibilities before nicotine exposure, the current data suggest that they may reflect predispositional individual differences. That our data-driven, whole-brain approach identified, with no a priori regional assumptions, a similar key role of the insula in nicotine dependence, supports its investigation for predicting future smoking behavior risk and early interventions in humans. A recent study from our laboratory using data-driven graph theory analyses observed changes in network connectivity including insular-based circuits driven by individual differences in nicotine metabolic rate (Li et al., 2017), once again suggesting that these circuits and networks change as a function of differential nicotine exposure.

\section{Insular-frontal module connectivity may bias salience attribution}

The brain-behavior relationships between insular-frontal connectivity and development of and reversal from nicotine dependence followed similar patterns: greater connectivity strength correlated with greater dependence severity, while weaker connectivity was related to enhanced dependence reversal following abstinence. Given the role of the insula and frontal cortical regions in processing internal and external environmental representations to guide decisions and behavioral output (Craig, 2009), we interpret these findings as intrinsic differences in saliency attribution. Heightened insular-frontal connectivity may bias stimuli processing toward the most homeostatically relevant salient events, whether internally (via striatal circuits) or externally (via sensory association cortices) generated. Thus, insularfrontal connectivity may follow an inverted " $U$ " pattern of optimal connectivity - too little confers minimal processing of stimuli while excess confers misattribution of saliency, disadvantageously biasing salience attribution. This theoretical framework of optimal insular connectivity is supported by research demonstrating hyperconnectivity and hypoconnectivity of the SN in patients with various neuropsychiatric conditions (Uddin, 2015). Notably, this interpretation of saliency bias is a working theory and was not directly tested herein; baseline behavioral tests of salience attribution and/or cue reactivity are necessary to validate our interpretation. Nevertheless, this putative nicotine dependence risk biomarker offers the exciting possibility of interrogating these networks for targeted smoking prevention and assessing cessation treatments.

\section{Limitations and future directions}

The observed brain-behavior relationships are correlational, and future preclinical studies should determine the causal influence of this insular-frontal circuit on dependence development, for example, by modulating its connections before nicotine exposure and observing the resultant effect on dependence susceptibility. Environmental and/or experiential perturbations known to influence the response to drugs of abuse should be tested for their effects on insular connectivity and its predictive capacity for dependence severity. Our assessment of dependence and method of nicotine administration were, like all models, imperfect: we captured the effects of noncontingent nicotine on only one facet of nicotine dependence. Here, mecamylamine-precipitated withdrawal was considered as a surrogate of nicotine dependence severity, given its relationship with dose (Malin et al., 1992) and intake in rodents (O'Dell et al., 2007). It is noteworthy that in smokers, while mecamylamine does not precipitate physical withdrawal, importantly it does increase nicotine intake (Nemeth-Coslett et al., 1986; Rose et al., 2001) and craving (Eissenberg et al., 1996). Thus, the withdrawal precipitated by mecamylamine in humans and rodents is related to the level of nicotine dependence and can promote active nicotine intake, supporting its use herein as a proxy for dependence. Finally, only male rats were included in this study. Future work necessitates using both sexes and additional behavioral models, including nicotine self-administration/reinstatement models coupled with assays of affective withdrawal.

\section{Conclusions}

To our knowledge, this is the first study to demonstrate the ability of brain connectivity before drug exposure to predict risk and/or resilience to developing nicotine dependence. With the pervasive individual and societal burden of nicotine addiction (Surgeon General, 2014) and the high relapse rates for those who attempt to quit (Chaiton et al., 2016), early identification of vulnerable individuals before the conversion from drug experimentation to dependence is an important goal to understand, prevent, and/or minimize the consequences of smoking. While putative neuroimaging biomarkers based on cross-sectional studies of drug exposure and dependence severity have been identified for nicotine addiction (Fedota and Stein, 2015), practical constraints of collecting longitudinal neuroimaging data from humans have thus far precluded the development of predictive biomarkers; data currently being collected from the ABCD study (Bjork et al., 2017) are expected to provide such insights. The preclinical results presented here offer a novel finding of an insular-frontal cortical-based predictive biomarker of addiction severity and recovery and, if instantiated in humans, such biomarkers could identify those individuals most at risk to develop dependence 
and, importantly, those who might best recover from nicotine dependence following treatment.

\section{References}

Ahmed SH, Lenoir M, Guillem K (2013) Neurobiology of addiction versus drug use driven by lack of choice. Curr Opin Neurobiol 23:581-587.

Ash JA, Lu H, Taxier LR, Long JM, Yang Y, Stein EA, Rapp PR (2016) Functional connectivity with the retrosplenial cortex predicts cognitive aging in rats. Proc Natl Acad Sci U S A 113:12286-12291.

Belin D, Belin-Rauscent A, Everitt BJ, Dalley JW (2016) In search of predictive endophenotypes in addiction: insights from preclinical research. Genes Brain Behav 15:74-88.

Belin-Rauscent A, Daniel ML, Puaud M, Jupp B, Sawiak S, Howett D, McKenzie C, Caprioli D, Besson M, Robbins TW, Everitt BJ, Dalley JW, Belin D (2016) From impulses to maladaptive actions: the insula is a neurobiological gate for the development of compulsive behavior. Mol Psychiatry 21:491-499.

Benwell ME, Balfour DJ, Birrell CE (1995) Desensitization of the nicotineinduced mesolimbic dopamine responses during constant infusion with nicotine. Br J Pharmacol 114:454-460.

Bierut LJ, Stitzel JA, Wang JC, Hinrichs AL, Grucza RA, Xuei X, Saccone NL, Saccone SF, Bertelsen S, Fox L, Horton WJ, Breslau N, Budde J, Cloninger CR, Dick DM, Foroud T, Hatsukami D, Hesselbrock V, Johnson EO, Kramer J, et al (2008) Variants in nicotinic receptors and risk for nicotine dependence. Am J Psychiatry 165:1163-1171.

Bjork JM, Straub LK, Provost RG, Neale MC (2017) The ABCD study of neurodevelopment: identifying neurocircuit targets for prevention and treatment of adolescent substance abuse. Curr Treat Options Psychiatry 4:196-209.

Brynildsen JK, Najar J, Hsu LM, Vaupel DB, Lu H, Ross TJ, Yang Y, Stein EA (2016) A novel method to induce nicotine dependence by intermittent drug delivery using osmotic minipumps. Pharmacol Biochem Behav 142: $79-84$.

Brynildsen JK, Hsu LM, Ross TJ, Stein EA, Yang Y, Lu H (2017) Physiological characterization of a robust survival rodent fMRI method. Magn Reson Imaging 35:54-60.

Bullmore E, Sporns O (2009) Complex brain networks: graph theoretical analysis of structural and functional systems. Nat Rev Neurosci 10:186198.

Centers for Disease Control and Prevention (2018) Smoking is down, but almost 38 million American adults still smoke: cigarette smoking remains high among certain groups. Atlanta, GA: Centers for Disease Control and Prevention.

Chaiton M, Diemert L, Cohen JE, Bondy SJ, Selby P, Philipneri A, Schwartz R (2016) Estimating the number of quit attempts it takes to quit smoking successfully in a longitudinal cohort of smokers. BMJ Open 6:e011045.

Clewett D, Luo S, Hsu E, Ainslie G, Mather M, Monterosso J (2014) Increased functional coupling between the left fronto-parietal network and anterior insula predicts steeper delay discounting in smokers. Hum Brain Mapp 35:3774-3787.

Cox RW (1996) AFNI: software for analysis and visualization of functional magnetic resonance neuroimages. Comput Biomed Res Int J 29:162-173.

Craig AD (2009) How do you feel-now? The anterior insula and human awareness. Nat Rev Neurosci 10:59-70.

Damaj MI, Kao W, Martin BR (2003) Characterization of spontaneous and precipitated nicotine withdrawal in the mouse. J Pharmacol Exp Ther 307:526-534.

Eissenberg T, Griffiths RR, Stitzer ML (1996) Mecamylamine does not precipitate withdrawal in cigarette smokers. Psychopharmacology (Berl) 127: $328-336$.

Epping-Jordan MP, Watkins SS, Koob GF, Markou A (1998) Dramatic decreases in brain reward function during nicotine withdrawal. Nature 393: $76-79$.

Fedota JR, Stein EA (2015) Resting-state functional connectivity and nicotine addiction: prospects for biomarker development. Ann N Y Acad Sci 1349:64-82.

Fedota JR, Matous AL, Salmeron BJ, Gu H, Ross TJ, Stein EA (2016) Insula demonstrates a non-linear response to varying demand for cognitive control and weaker resting connectivity with the executive control network in smokers. Neuropsychopharmacology 41:2557-2565.

Fedota JR, Ding X, Matous AL, Salmeron BJ, McKenna MR, Gu H, Ross TJ, Stein EA (2018) Nicotine abstinence influences the calculation of sa- lience in discrete insular circuits. Biol Psychiatry Cogn Neurosci Neuroimaging 3:150-159.

Flynn FG (1999) Anatomy of the insula functional and clinical correlates. Aphasiology 13:55-78.

Guimerà R, Nunes Amaral LA (2005) Functional cartography of complex metabolic networks. Nature 433:895-900.

He Y, Wang J, Wang L, Chen ZJ, Yan C, Yang H, Tang H, Zhu C, Gong Q, Zang Y, Evans AC (2009) Uncovering intrinsic modular organization of spontaneous brain activity in humans. PLoS One 4:e5226.

Hong LE, Gu H, Yang Y, Ross TJ, Salmeron BJ, Buchholz B, Thaker GK, Stein EA (2009) Association of nicotine addiction and nicotine's actions with separate cingulate cortex functional circuits. Arch Gen Psychiatry 66:431-441.

Hong LE, Hodgkinson CA, Yang Y, Sampath H, Ross TJ, Buchholz B, Salmeron BJ, Srivastava V, Thaker GK, Goldman D, Stein EA (2010) A genetically modulated, intrinsic cingulate circuit supports human nicotine addiction. Proc Natl Acad Sci U S A 107:13509-13514.

Hsu LM, Liang X, Gu H, Brynildsen JK, Stark JA, Ash JA, Lin CP, Lu H, Rapp PR, Stein EA, Yang Y (2016) Constituents and functional implications of the rat default mode network. Proc Natl Acad Sci USA 113:E4541-E4547.

Kobayashi M (2011) Macroscopic connection of rat insular cortex: anatomical bases underlying its physiological functions. Int Rev Neurobiol 97: 285-303.

Kolb B (1984) Functions of the frontal cortex of the rat: a comparative review. Brain Res 320:65-98.

Koob GF, Volkow ND (2016) Neurobiology of addiction: a neurocircuitry analysis. Lancet Psychiatry 3:760-773.

Koob GF, Maldonado R, Stinus L (1992) Neural substrates of opiate withdrawal. Trends Neurosci 15:186-191.

Liang X, He Y, Salmeron BJ, Gu H, Stein EA, Yang Y (2015) Interactions between the salience and default-mode networks are disrupted in cocaine addiction. J Neurosci 35:8081-8090.

Liang X, Hsu LM, Lu H, Sumiyoshi A, He Y, Yang Y (2018) The rich-club organization in rat functional brain network to balance between communication cost and efficiency. Cereb Cortex 28:924-935.

Li S, Yang Y, Hoffmann E, Tyndale RF, Stein EA (2017) CYP2A6 genetic variation alters striatal-cingulate circuits, network hubs, and executive processing in smokers. Biol Psychiatry 81:554-563.

Liu M, Jiang Y, Wedow R, Li Y, Brazel DM, Chen F, Datta G, DavilaVelderrain J, McGuire D, Tian C, Zhan X, Zhan X, Zhan X, Choquet H, Docherty AR, Faul JD, Foerster JR, Fritsche LG, Gabrielsen ME, Gordon SD, et al (2019) Association studies of up to 1.2 million individuals yield new insights into the genetic etiology of tobacco and alcohol use. Nat Genet 51:237-244.

Lu H, Scholl CA, Zuo Y, Demny S, Rea W, Stein EA, Yang Y (2010) Registering and analyzing rat fMRI data in the stereotaxic framework by exploiting intrinsic anatomical features. Magn Reson Imaging 28:146-152.

Lu H, Zou Q, Gu H, Raichle ME, Stein EA, Yang Y (2012) Rat brains also have a default mode network. Proc Natl Acad Sci U S A 109:3979-3984.

Malin DH (2001) Nicotine dependence: studies with a laboratory model. Pharmacol Biochem Behav 70:551-559.

Malin DH, Lake JR, Newlin-Maultsby P, Roberts LK, Lanier JG, Carter VA, Cunningham JS, Wilson OB (1992) Rodent model of nicotine abstinence syndrome. Pharmacol Biochem Behav 43:779-784.

Maslov S, Sneppen K (2002) Specificity and stability in topology of protein networks. Science 296:910-913.

Menon V, Uddin LQ (2010) Saliency, switching, attention and control: a network model of insula function. Brain Struct Funct 214:655-667.

Morales AM, Ghahremani D, Kohno M, Hellemann GS, London ED (2014) Cigarette exposure, dependence, and craving are related to insula thickness in young adult smokers. Neuropsychopharmacology 39:1816-1822.

Naqvi NH, Rudrauf D, Damasio H, Bechara A (2007) Damage to the insula disrupts addiction to cigarette smoking. Science 315:531-534.

Nemeth-Coslett R, Henningfield J, O’Keeffe M, Griffiths R (1986) Effects of mecamylamine on human cigarette smoking and subjective ratings. Psychopharmacology (Berl) 88:420-425.

Newman ME (2004) Analysis of weighted networks. Phys Rev E Stat Nonlin Soft Matter Phys 70:056131.

Newman ME (2006) Modularity and community structure in networks. Proc Natl Acad Sci U S A 103:8577-8582.

Nomi JS, Farrant K, Damaraju E, Rachakonda S, Calhoun VD, Uddin LQ 
(2016) Dynamic functional network connectivity reveals unique and overlapping profiles of insula subdivisions. Hum Brain Mapp 37: $1770-1787$

O’Dell LE, Chen SA, Smith RT, Specio SE, Balster RL, Paterson NE, Markou A, Zorrilla EP, Koob GF (2007) Extended access to nicotine selfadministration leads to dependence: circadian measures, withdrawal measures, and extinction behavior in rats. J Pharmacol Exp Ther 320:180-193.

Palmatier MI, Peterson JL, Wilkinson JL, Bevins RA (2004) Nicotine serves as a feature-positive modulator of Pavlovian appetitive conditioning in rats. Behav Pharmacol 15:183-194.

Paxinos G, Watson C (2007) The rat brain in stereotaxic coordinates. New York: Academic.

Perry DC, Xiao Y, Nguyen HN, Musachio JL, Dávila-García MI, Kellar KJ (2002) Measuring nicotinic receptors with characteristics of $\alpha 4 \beta 2, \alpha 3 \beta 2$ and $\alpha 3 \beta 4$ subtypes in rat tissues by autoradiography. J Neurochem 82:468-481.

Piasecki TM, Fiore MC, Baker TB (1998) Profiles in discouragement: two studies of variability in the time course of smoking withdrawal symptoms. J Abnorm Psychol 107:238-251.

Piasecki TM, Niaura R, Shadel WG, Abrams D, Goldstein M, Fiore MC, Baker TB (2000) Smoking withdrawal dynamics in unaided quitters. J Abnorm Psychol 109:74-86.

Picard F, Sadaghiani S, Leroy C, Courvoisier DS, Maroy R, Bottlaender M (2013) High density of nicotinic receptors in the cingulo-insular network. Neuroimage 79:42-51.

Power JD, Cohen AL, Nelson SM, Wig GS, Barnes KA, Church JA, Vogel AC, Laumann TO, Miezin FM, Schlaggar BL, Petersen SE (2011) Functional network organization of the human brain. Neuron 72:665-678.

Power JD, Schlaggar BL, Lessov-Schlaggar CN, Petersen SE (2013) Evidence for hubs in human functional brain networks. Neuron 79:798 -813.

Rezvani AH, Levin ED (2001) Cognitive effects of nicotine. Biol Psychiatry 49:258-267.

Rose JE, Behm FM, Westman EC (2001) Acute effects of nicotine and mecamylamine on tobacco withdrawal symptoms, cigarette reward and ad lib smoking. Pharmacol Biochem Behav 68:187-197.

Saccone SF, Hinrichs AL, Saccone NL, Chase GA, Konvicka K, Madden PA, Breslau N, Johnson EO, Hatsukami D, Pomerleau O, Swan GE, Goate AM, Rutter J, Bertelsen S, Fox L, Fugman D, Martin NG, Montgomery GW, Wang JC, Ballinger DG, et al (2007) Cholinergic nicotinic receptor genes implicated in a nicotine dependence association study targeting 348 candidate genes with 3713 SNPs. Hum Mol Genet 16:36-49.

Sherva R, Wilhelmsen K, Pomerleau CS, Chasse SA, Rice JP, Snedecor SM, Bierut LJ, Neuman RJ, Pomerleau OF (2008) Association of a single nucleotide polymorphism in neuronal acetylcholine receptor subunit alpha 5 (CHRNA5) with smoking status and with "pleasurable buzz" during early experimentation with smoking. Addiction 103:1544-1552.

Sporns O (2013) Network attributes for segregation and integration in the human brain. Curr Opin Neurobiol 23:162-171.

Surgeon General (2014) The health consequences of smoking — 50 years of progress: a report of the surgeon general, 2014. Washington, DC: U.S. Department of Health and Human Services.

Sutherland MT, Carroll AJ, Salmeron BJ, Ross TJ, Hong LE, Stein EA (2013) Down-regulation of amygdala and insula functional circuits by varenicline and nicotine in abstinent cigarette smokers. Biol Psychiatry 74:538 546.

Sutherland MT, Riedel MC, Flannery JS, Yanes JA, Fox PT, Stein EA, Laird AR (2016) Chronic cigarette smoking is linked with structural alterations in brain regions showing acute nicotinic drug-induced functional modulations. Behav Brain Funct 12:16.

Tunstall BJ, Kearns DN (2015) Sign-tracking predicts increased choice of cocaine over food in rats. Behav Brain Res 281:222-228.

Uddin LQ (2015) Salience processing and insular cortical function and dysfunction. Nat Rev Neurosci 16:55-61.

Vann RE, Balster RL, Beardsley PM (2006) Dose, duration, and pattern of nicotine administration as determinants of behavioral dependence in rats. Psychopharmacology (Berl) 184:482-493.

Vaudano E, Legg CR, Glickstein M (1991) Afferent and efferent connections of temporal association cortex in the rat: a horseradish peroxidase study. Eur J Neurosci 3:317-330.

Villafuerte S, Heitzeg MM, Foley S, Yau WY, Majczenko K, Zubieta JK, Zucker RA, Burmeister M (2012) Impulsiveness and insula activation during reward anticipation are associated with genetic variants in GABRA2 in a family sample enriched for alcoholism. Mol Psychiatry 17:511-519.

Watkins SS, Stinus L, Koob GF, Markou A (2000) Reward and somatic changes during precipitated nicotine withdrawal in rats: centrally and peripherally mediated effects. J Pharmacol Exp Ther 292:1053-1064.

Zhang X, Salmeron BJ, Ross TJ, Geng X, Yang Y, Stein EA (2011) Factors underlying prefrontal and insula structural alterations in smokers. Neuroimage 54:42-48. 